\title{
TESES E DISSERTAÇÕES BRASILEIRAS SOBRE BIM: UMA ANÁLISE DO PERÍODO DE 2013 A 2018
}

\section{BRAZILIAN THESIS AND DISSERTATIONS ON BIM: AN ANALYSIS OF THE PERIOD 2013 TO 2018}

\author{
Érica de Sousa Checcucci ${ }^{1}$ \\ Universidade Federal da Bahia, Salvador, BA, Brasil, erica.checcucci@ufba.br
}

\begin{abstract}
Resumo
Este trabalho identifica e analisa teses e dissertações disponíveis no Catálogo de teses e dissertações da CAPES que foram defendidas no Brasil entre 2013 e 2018 e tratam da Modelagem da Informação da Construção ou Building Information Modelling (BIM). Objetiva apresentar um panorama sobre os principais temas abordados nestas pesquisas, assim como identificar onde foram realizadas. Ainda, é feito um recorte sobre aqueles trabalhos que discutem questões sobre o ensino de BIM buscando contribuir com a implantação desta modelagem nos cursos relacionados com a Arquitetura, Engenharia, Construção e Operação de edificações (AECO). A Análise de Conteúdo foi o método de pesquisa qualitativa adotado para realizar tanto a seleção quanto a análise dos trabalhos. Dentre os resultados obtidos, destaca-se que: (a) já existem trabalhos sobre BIM nas diversas fases do ciclo de vida da edificação, mas a maioria deles trata da etapa de projetação; (b) $78,3 \%$ das pesquisas encontradas foram produzidas no eixo sul-sudeste do país e em instituições de ensino sediadas nas capitais e grandes cidades, indicando a necessidade de interiorização e difusão do BIM em outras regiões; (c) existe uma diversidade de temas já pesquisados relacionados com o BIM que encaminham questões sobre a modelagem e podem auxiliar na sua adoção tanto em instituições de ensino quanto no mercado de trabalho. Finalmente, este artigo contribui para adoção desta modelagem no país, na medida em que sistematiza informações já disponíveis, auxiliando tanto aqueles que buscam se capacitar quanto aqueles que promovem formações ou pretendem trabalhar com BIM.
\end{abstract}

Palavras-chave: Modelagem da Informação da Construção. BIM. Teses e dissertações. Levantamento. Ensino-aprendizagem.

\begin{abstract}
This paper identifies and analyzes theses and dissertations available in the Catalog of theses and dissertations of CAPES that were defended in Brazil between 2013 and 2018 and deal with the Modeling of Construction Information. It aims to present an overview of the main topics covered in these studies, and identify where they were carried out. In addition, a study is made of those works that discuss BIM education, aiming to contribute to the implementation of this modelling in courses related to Architecture, Civil Engineering, Construction and Building Operations. Content Analysis was the qualitative research method adopted to perform both the selection and the analysis of the identified researches. Among the results, it is highlighted that: (a) there are already studies on BIM in the various phases of the life cycle of the building, but most of them deal with the design stage; (b) $78.3 \%$ of the studies were produced in the south and southeast regions of the country and in educational institutions based in capitals and large cities, indicating the need for internalization and diffusion of BIM to other places; (c) there are a variety of researched topics related to the subject that address questions about BIM and can assist in its adoption in both educational institutions and the labor market. Finally, this work contributes to the adoption of BIM in Brazil as it systematizes information that is already available, helping both those who seek training and those who promote training or intend to work in this field.
\end{abstract}

Keywords: Building Information Modelling. BIM. Theses and dissertations. Survey. Teaching learning.

How to cite this article:

CHECCUCCI, Érica de Sousa. Teses e dissertações brasileiras sobre BIM: uma análise do período de 2013 a 2018. PARC Pesquisa em Arquitetura e Construção, Campinas, SP, v. 10, p. e019008, fev. 2019. ISSN 1980-6809. Disponível em: <https://periodicos.sbu.unicamp.br/ojs/index.php/parc/article/view/8653708>. Acesso em: 26 fev. 2019. doi:https://doi.org/10.20396/parc.v10i0.8653708. 


\section{Introdução}

A Modelagem da Informação da Construção ou Building Information Modelling (BIM) envolve um conjunto de tecnologias computacionais e métodos de trabalho que visam a representação completa da edificação através de modelos que darão suporte aos profissionais da Arquitetura, Engenharia, Construção e Operação (AECO) durante todo seu ciclo de vida: da concepção, passando pelo planejamento da construção, construção, operação (que envolve uso, gestão e manutenção) e reciclagem ou demolição, quando encerrar sua vida útil.

Dada sua abrangência e complexidade, é possível encontrar diferentes definições de BIM - umas são mais amplas, enquanto outras ressaltam alguns de seus aspectos. Por exemplo, Succar (2009), Ruschel e colaboradores (2011) e Kassem (2018), dentre outros, conceituam BIM a partir da combinação de políticas (de difusão, padronização, normativas, etc.); de processos (para desenvolvimentos de modelos, componentes, etc.) e tecnologias (conjunto de hardware e software). Já Scheer e Ayres Filho (2009) explicam BIM a partir de 4 níveis de modelagem: (1) a supermodelagem, que trata de processos e mecanismos de colaboração; (2) a metamodelagem, que discute padrões de arquivos para a troca de informações entre os modelos; (3) a modelagem, que trata da produção dos modelos e, (4) a micromodelagem, que diz respeito a criação dos componentes que serão instanciados na modelagem.

Vantagens do BIM já estão amplamente atestadas na literatura nacional e internacional (KYMMELL, 2008; KIM 2012; CAIXETA, 2013; BERNSTEIN et al., 2014; CHECCUCCl, 2014; SUCCAR; KASSEM, 2015; SANTOS; LIMA; CAMPOS, 2017). No entanto, ainda que já existam experiências de sucesso no uso desta modelagem no país, está claro que a sua adoção no Brasil se encontra em estágios iniciais, tanto no mercado da Construção Civil quanto nos cursos de formação de profissionais da área, sejam eles em nível tecnológico, de graduação ou pós-graduação (RUSCHELL; ANDRADE; MORAIS, 2013; ITO; SCHEER, 2017).

Uma perspectiva animadora para ampliação da adoção do BIM no país surgiu com a publicação do Decreto 9.377 de 17 de maio de 2018 (BRASIL, 2018), que instituiu a Estratégia Nacional de Disseminação do Building Information Modelling, e que, a partir de 9 objetivos específicos, busca promover condições para investimento e difusão desta modelagem no Brasil.

Dentro deste contexto, este trabalho surge motivado pelo Encontro Nacional de Ensino de BIM (ENEBIM), que aconteceu em Campinas entre 17 a 19 de setembro de 2018 e reuniu a comunidade científica, docentes, instrutores e especialistas em torno de discussões sobre o ensino e aprendizagem desta modelagem. Neste evento, ficou clara a necessidade de se fazer um esforço coletivo objetivando capacitar mão de obra qualificada para dar conta dos desafios que o BIM apresenta. Diante disto, objetivando sistematizar informações já disponíveis sobre o tema para auxiliar na adoção do BIM, tanto no mercado de trabalho quanto nas instituições de ensino, foi realizada uma busca no Catálogo de teses e dissertações da CAPES ${ }^{1}$ para identificar trabalhos que tratam desta modelagem e que foram publicados entre 2013 e $2018^{2}$.

A Análise de Conteúdo (BARDIN, 1979) foi o método de pesquisa qualitativa escolhido para realizar a seleção e avaliação dos trabalhos e criar o panorama aqui apresentado. A sua grande vantagem é ajudar a identificar e agrupar elementos diferentes entre si a partir de categorias conceituais previamente definidas. Uma característica que deve ser ressaltada neste método é que ele envolve a subjetividade do pesquisador, responsável por escolher de que forma os dados serão classificados e agrupados. Como afirmou 
Bardin (1979, p. 37), “... tudo depende, no momento da escolha dos critérios de classificação, daquilo que se procura ou que se espera encontrar".

No entanto, apesar de envolver certa subjetividade, a pesquisa qualitativa possui um rigor próprio que pauta-se em:

[...] amostras intencionais, construídas pelo critério de consistência informada, justificada e explicitada pelo pesquisador e sua comunidade em torno da sua pertinência e relevância para responder as questões de pesquisa, [...] (MACEDO, 2009, p. 96, grifo do autor).

Esta pesquisa busca responder as seguintes perguntas, relacionadas com BIM: (a) quais são as áreas de estudo mais escolhidas para serem aprofundadas nas teses e dissertações brasileiras?; (b) Dentro do ciclo de vida da edificação, quais são as fases mais estudadas e quais são as que demandam mais estudos para encaminhar questões ainda em aberto sobre a modelagem?; (c) Quais são e onde ficam as instituições de ensino onde estes trabalhos são realizados?

\section{A adoção de BIM no Brasil}

A modelagem da informação da construção ainda está em uma fase inicial de implantação no país, principalmente considerando seu território continental, com seus 26 estados, mais de 5.500 municípios e o Distrito Federal.

Checcucci, Pereira e Amorim (2013) identificaram em 2013 que o estágio de implantação do BIM no Brasil era inicial, afirmando encontrar experiências de sua utilização no projeto e construção da edificação, mas não nas etapas de operação, manutenção e requalificação ou demolição da edificação. Anos depois, Mota e Ruschel (2016; 2017) constataram um cenário similar, e afirmaram que dentro do ciclo de vida da edificação, BIM era adotado nas fases de projeto e construção, mas na fase de operação da edificação ainda estava no início.

Brocardo e Scheer (2017) afirmam que algumas empresas começaram a adotar BIM no início dos anos 2000 mas que poucas conseguiram utilizar a modelagem no processo completo de projeto. Ainda, Machado, Ruschel e Scheer (2017) constatam que a pesquisa sobre BIM no Brasil está mais avançada do que sua implantação nas empresas ligadas à Construção Civil.

Especificamente em relação ao ensino, Ruschel, Andrade e Morais (2013) identificaram que algumas universidades já tinham experiências de adoção de BIM. No entanto, estes autores avaliaram que a implantação estava sendo feita de forma gradual e pouco eficiente. No ano seguinte, $\mathrm{CHECCUCCI}$ (2014) também identificou algumas experiências realizadas no país, mas ainda em poucas universidades, de modo pontual e em uma ou duas disciplinas da matriz curricular. A formação de nível técnico foi discutida por Cruz, Cuperschmid e Ruschel (2017) que acompanharam a inserção de BIM no curso Técnico em Edificações da escola Orlando Lavieiro Ferraiuolo, do SENAI em São Paulo.

Recentemente, em Campinas, foram discutidas experiências de ensino e aprendizagem vivenciadas em diferentes partes do Brasil (ENEBIM, 2018; CBIC, 2018). Percebeu-se que muitas propostas de qualidade estão sendo realizadas para formação tanto em nível técnico, quanto de graduação e/ou pós-graduação. Neste evento científico, pode-se constatar que a maioria das experiências apresentadas: (a) acontecem a partir de um esforço pessoal dos docentes envolvidos com o componente curricular, que se articulam com colegas para viabilizar as disciplinas; (b) acontecem de forma pontual nos currículos dos cursos; (c) continuam abrangendo as fases iniciais do ciclo de vida da 
edificação. Também ficou clara a demanda por docentes capacitados em BIM para dar conta do desafio de formação existente no Brasil.

No entanto, já existem muitas pesquisas de pós-graduação que encaminham questões ou propõem alternativas para auxiliar na adoção do BIM, tanto no ensino, quanto no mercado de trabalho. Diante disto, o trabalho aqui apresentado seleciona este material já disponível e o classifica, de forma a colaborar na sua utilização por todos aqueles envolvidos com o tema.

\section{Pesquisas que analisaram a produção científica nacional sobre BIM}

Andrade e Ruschel (2009) encontraram, classificaram e analisaram 13 artigos publicados sobre o tema em eventos científicos no Brasil e constataram que BIM ainda era pouco estudado no país naquela época, com as pesquisas ainda incipientes e enfocando mais sobre o processo de projeto de forma isolada nos escritórios de arquitetura. Utilizando a análise de conteúdo, os autores organizaram os trabalhos em 4 categorias distintas de acordo com o enfoque das pesquisas: (a) nos conceitos, vantagens e desafios do BIM; (b) no uso de BIM no processo de projeto; (c) na colaboração e interoperabilidade entre aplicativos; e, (d) nas estratégias para customizar aplicativos BIM (ANDRADE; RUSCHEL, 2009).

Dois anos depois, Checcucci, Pereira e Amorim (2011) apresentaram um panorama das publicações nacionais sobre o tema a partir de buscas em anais de eventos científicos, revistas eletrônicas e bases de dados nacionais abrangendo um lapso temporal de 2005 a 2010. Os autores identificaram, classificaram e analisaram uma amostra de 32 artigos, 10 dissertações e 2 teses sobre BIM, totalizando 44 trabalhos avaliados. Também utilizaram a análise de conteúdo para organizar a amostra encontrada nas 4 categorias propostas por Andrade e Ruschel (2009), acrescentando mais uma quinta categoria que agrupou pesquisas com enfoque na adoção de BIM no ensino. Encontraram um cenário ainda inicial nas investigações, apesar de identificarem um aumento na quantidade das produções. Ressaltaram que: (a) os pesquisadores concentravam-se nas regiões Sul e Sudeste do país e, portanto, era necessária a criação ou ampliação de redes de pesquisas; (b) já se identificava a preocupação da academia em inserir BIM no ensino, com 9 artigos tratando de experiências já realizadas; (c) havia pouco material de acesso livre sobre BIM, com algumas teses e dissertações não publicadas no banco de teses da Capes nem no Portal Domínio Públicos e os anais de alguns importantes eventos científicos não disponíveis na internet (CHECCUCCI; PEREIRA; AMORIM, 2011).

Alguns anos depois, Machado, Ruschel e Scheer (2017) apresentaram uma ampla e profunda análise da produção científica nacional sobre o tema. Os autores avaliaram uma amostra de 405 trabalhos, sendo 278 artigos publicados em anais de eventos científicos, 52 em periódicos especializados, 63 dissertações de mestrado e 12 teses de doutorado. Abrangendo um intervalo temporal de 16 anos (de 2000 a 2015), os autores: (a) discutiram a evolução da produção bibliográfica nacional e da formação de recursos humanos; (b) identificaram cientistas com maior número de publicações e fizeram um levantamento quantitativo de sua produção; (c) identificaram a formação de pesquisadores por região e instituição; $(d)$ analisaram as palavras-chave mais utilizadas nas publicações; (e) identificaram e classificaram os métodos científicos empregados nas teses e dissertações (MACHADO; RUSCHEL; SCHEER, 2017).

Dentre as constatações realizadas por Machado, Ruschel e Scheer (2017) pode ser destacado: (a) o crescimento de publicações em anais de eventos, notadamente a partir de 2010 e em periódicos a partir de 2012; (b) a baixa cooperação científica entre 
instituições nacionais e internacionais nas publicações; (c) o interesse em pesquisar questões relacionadas com a interoperabilidade e processo de projeto, tanto na arquitetura quanto na construção civil; (d) o destaque da USP, UFPR e UNB no número de teses e dissertações defendidas.

Os autores observaram que o conhecimento construído não está sendo devidamente difundido, ao contrastar o número de 52 trabalhos publicados em periódicos com as 75 teses e dissertações defendidas no período analisado. Também constataram que nos últimos anos houve um decréscimo nas defesas de mestrado e crescimento nas titulações de doutorado, o que os levou a inferir que existe uma maturidade e evolução na formação acadêmica com os mestres titulando-se doutores. Esta maturidade pode ser também verificada pelo aumento do número de publicações em periódicos (MACHADO; RUSCHEL; SCHEER, 2017).

A pesquisa aqui apresentada enfoca os trabalhos de pós-graduação defendidos e publicados entre 2013 e 2018. Diante disto, possui uma amostra de análise composta apenas por teses e dissertações. Apesar de menos amplo no tipo de trabalho que avalia, não contemplando artigos publicados ou outras comunicações científicas, debruça-se com mais cuidado sobre os temas relacionados com BIM que são discutidos nas pósgraduações stricto-senso do Brasil, apresentando um panorama atualizado deste contexto.

\section{O método de pesquisa}

Este trabalho pode ser classificado como exploratório, uma vez que utiliza procedimentos sistemáticos para coleta e análise de dados, gerando descrições quantitativas e qualitativas da amostra estudada (TRIPODI et al., 1975 apud MARCONI; LAKATO, 2003, p. 187).

Os dados foram coletados nos documentos disponíveis no Catálogo de teses e dissertações da CAPES, selecionados e analisados com auxílio da Análise de Conteúdo (BARDIN, 1970) seguindo as seguintes etapas:

(a) busca por 3 termos: BIM, "Building Information Modelling" e "Modelagem da Informação da Construção" nas 4 grandes áreas de conhecimento (Ciências exatas e da terra; Ciências Sociais Aplicadas; Engenharia e, Multidisciplinar) e em 8 áreas específicas de conhecimento (Administração pública; Arquitetura e Urbanismo; Construção Civil; Engenharia Civil; Engenharia de Produção; Engenharia, tecnologia e gestão; Sociais e humanidades e, Tecnologia de Arquitetura e Urbanismo);

(b) para cada termo, os trabalhos foram classificados segundo diferentes categorias de pós-graduação existentes na base de dados (doutorado, mestrado e mestrado profissional) e agrupados por ano, considerando o período entre 2013 e 2018;

(c) foram comparadas as listas obtidas para cada termo, de forma a identificar trabalhos repetidos e unificá-las;

(d) os títulos dos trabalhos da lista unificada (organizados por categoria de pósgraduação e ano) foram lidos e desta forma pôde-se confirmar sua pertinência em relação ao tema BIM e identificar categorias conceituais para agrupá-los. Quando havia dúvida se o trabalho de fato tratava de BIM, o resumo, palavras-chave, sumário e, às vezes, a conclusão da pesquisa eram lidos. Também se fazia uma busca em todo o texto pelos 3 termos consultados na etapa (a) para avaliar sua prevalência e aumentar a assertividade sobre manter ou não o trabalho na análise. Nesta etapa foram retirados 9 trabalhos identificados pelo método de busca mas 
que não tinham relação com o tema ou não tinham BIM como foco da investigação (por exemplo, o trabalho de um autor cujo sobrenome é BIM mas que não trata deste tema e um trabalho que citava BIM apenas uma vez em todo o texto).

(e) os trabalhos selecionados foram classificados em categorias e os resultados encontrados foram sistematizados e avaliados.

(f) foi realizada uma análise com maior profundidade sobre os trabalhos que tratam do ensino e aprendizagem de BIM, buscando identificar, nesta categoria, quais as maiores contribuições dos autores destas pesquisas.

\section{Resultados obtidos}

\section{A amostra final}

Após a realização das buscas na base de dados, foram encontrados 152 trabalhos. No entanto, ao verificar a pertinência deles em relação ao tema BIM - etapa (e) - foram retirados 9 trabalhos, totalizando 143 pesquisas, sendo 19 teses, 105 dissertações de mestrado e 19 de mestrado profissional publicadas no período definido, como ilustrado pelo Gráfico 1.

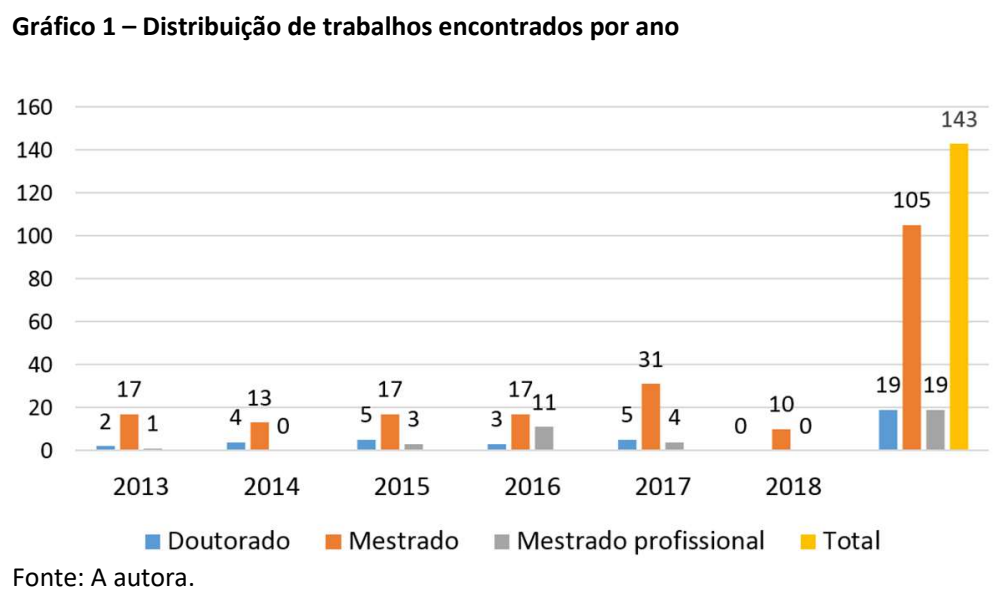

\section{Categorias de investigação em BIM}

Diante da variedade dos temas pesquisados nos trabalhos encontrados, foram criadas 9 categorias conceituais para agrupa-los:

(a) Ensino: aqueles que tratam de disciplinas que adotam a modelagem; experiências de implantação em cursos e outras questões relacionadas com o ensino e aprendizagem de BIM.

(b) Avaliação do BIM: pesquisas que discutem vantagens e desvantagens da modelagem; de suas ferramentas; interoperabilidade entre programas; fazem comparativos com métodos tradicionais de trabalho e uso de ferramentas CAD; estudam adoção no mercado da Construção Civil, etc.

(c) Implantação: trabalhos que tratam de adoção de BIM em empresas; formas de contratação; Integrated Project Delivery (IPD) e temas correlatos.

(d) Projetação: trabalhos que tratam desta etapa do ciclo de vida da edificação. Dentro desta categoria, os textos foram, ainda, classificados como:

- (d.1) Gestão: pesquisas que discutem gestão, coordenação, compatibilização e/ou colaboração multidisciplinar no projeto. 
- (d.2) Conforto e sustentabilidade: aqueles que tratam de questões projetuais relacionadas com a melhoria do ambiente construído, redução de resíduos, de gasto energético, otimização da edificação, etc.

- (d.3) Aplicação: os que tratam de experiências em projetos específicos. Foram encontrados trabalhos que discutem adoção de BIM em projetos de arquitetura, infraestrutura, estrutura, saneamento, habitação de interesse social, projeto hidráulico, aeroportuário, dentre outros.

(e) Obra: trabalhos que tratam do planejamento, da gestão, do controle da construção e/ou BIM 4D;

(f) Orçamentação: pesquisas que tratam de extração de quantitativos, definição de orçamento de obra e/ou BIM 5D.

(g) Usos por terceiros: pesquisas que tratam do uso do BIM por entidades públicas ou de avaliação para, por exemplo, analisar o projeto para licitação; aprovar em órgãos públicos; obter certificação ambiental; fiscalizar obras; dentre outros.

(h) FM: trabalhos que discutem questões relacionadas com o pós-obra, gestão, operação, manutenção da edificação e/ou Facility Management (FM). E, finalmente,

(i) BIM + : Nesta categoria foram classificados trabalhos centrados na discussão da relação ou integração de BIM com outras tecnologias, como Realidade Aumentada (RA) ou Virtual (RV); modelos de nuvens de pontos; Internet das Coisas (IoT); sistemas generativos de modelagem geométrica; fabricação digital, dentre outros.

O Gráfico 2 ilustra a quantidade de trabalhos encontrados em cada categoria, separados entre os 3 tipos de pós-graduação: doutorado, mestrado e mestrado profissional. Foi ordenado de forma decrescente em relação ao total de trabalhos em cada categoria de forma a destacar os temas mais e menos pesquisados.

Gráfico 2 - Numero de trabalhos $\mathrm{x}$ categorias

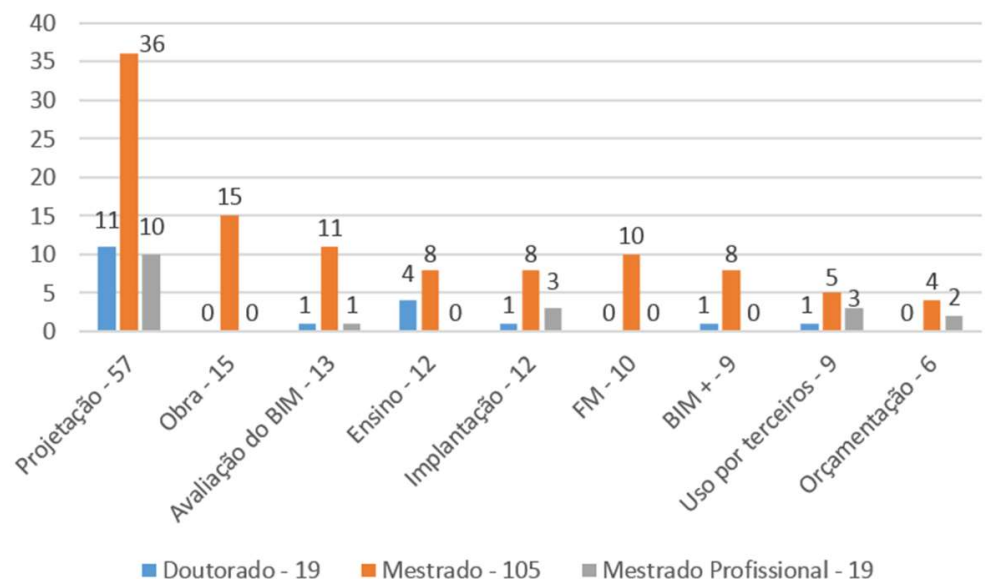

Fonte: A autora.

O Gráfico 3 mostra o resultado da análise efetuada nos 57 trabalhos que tratam da projetação, categorizando-os nas 3 subcategorias conceituais (gestão do projeto; conforto e sustentabilidade e, aplicação). 
Gráfico 3 - trabalhos que discutem BIM na etapa de projetação

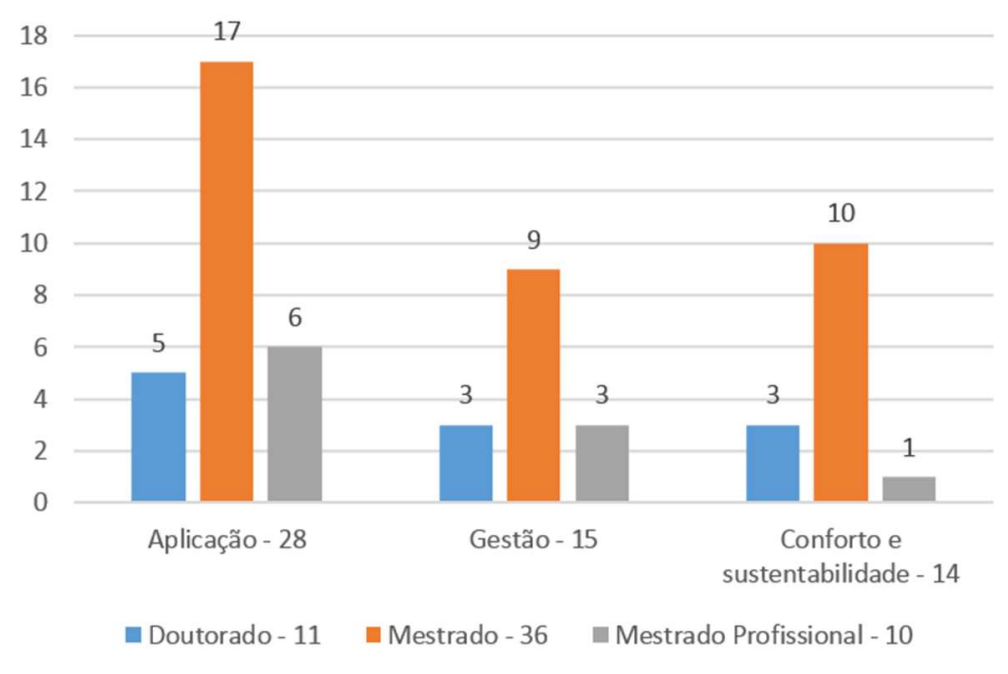

Fonte: A autora.

Dos 28 trabalhos classificados na categoria “Projetação / Aplicação”, que discutem BIM a partir de uma adoção em um projeto concreto, foi identificado que 13 deles são da área de projeto arquitetônico; 9 na área de estruturas (metálica - 4, de concreto armado - 4 e alvenaria estrutural - 1); 4 na área de projeto de instalações; 1 trata quantificação de resíduo de demolição e outro na área de projeto de infraestrutura.

Continuando as análises dos trabalhos encontrados, foi elaborado o Quadro 1, que identifica as instituições onde as 143 pesquisas foram realizadas, agrupando-as por Região e Estados do país e totalizado o número de trabalhos por Região, por Estado, por instituição e por categoria de pós-graduação. Como já apontado anteriormente por Checcucci, Pereira e Amorim (2011) e Machado, Ruschel e Scheer (2017), as regiões Sul e Sudeste do país destacam-se pela sua produção no tema. As instituições com maior número de defesas são a USP, UFPR, UFRGS, UNB e UNICAMP.

Finalmente, encerrando os resultados da busca e categorização das pesquisas, foram separadas aquelas que tratam especificamente sobre o ensino-aprendizagem de BIM, sobre as quais foi realizada uma análise mais aprofundada. A seção a seguir trata especialmente do resultado desta análise.

Teses e dissertações sobre ensino-aprendizagem de BIM³ - 2013 a 2018

Foram encontrados 12 trabalhos que tratam de ensino-aprendizagem de BIM no período investigado, sendo 4 de doutorado e 8 de mestrado, como ilustrado na Figura 1.

Caixeta (2013) discute as vantagens do BIM e as chances de suas ferramentas serem utilizadas para criar um novo paradigma de aprendizagem. Entre as conclusões do trabalho, pode ser destacado que o autor constata que existe demanda por professores capacitados em BIM e que os alunos que usam BIM compreendem melhor elementos estruturais do projeto e se preocupam mais com o processo de construção.

Checcucci (2014) desenvolve um método que pode ser aplicado para auxiliar na inserção de BIM nos currículos de cursos de graduação em engenharia civil e apresenta uma proposta de estruturação da área de Expressão Gráfica nestes cursos. Algumas conclusões deste trabalho podem ser citadas: (a) necessidade de atualização dos cursos e de docentes; (b) BIM favorece o aprendizado de conteúdos de engenharia; (c) é preciso que haja colaboração entre docentes e disciplinas; (d) deve-se trabalhar BIM em diversos componentes ao longo da formação do estudante e desde o início do curso; (e) 
CHECCUCCI, Érica de Sousa.

Teses e dissertações brasileiras sobre BIM: uma análise do período de 2013 a 2018

metodologias de ensino-aprendizagem ativas são mais adequadas para a formação em BIM. O método desenvolvido como produto desta tese auxilia a identificar interfaces entre um curso e BIM a partir da análise da estrutura curricular proposta. Ele foi detalhado por Checcucci e Amorim (2014), adaptado e aplicado por Cruz, Cuperschmid e Ruschel (2017) e por Rodrigues e Lima (2017).

Quadro 1 - análise dos locais onde os trabalhos foram realizados

\begin{tabular}{|c|c|c|c|c|c|c|c|}
\hline Região & Estado & Total/estado & IES & Total/IES & $\begin{array}{c}\text { Doutorado } \\
19\end{array}$ & $\begin{array}{c}\text { Mestrado } \\
105\end{array}$ & $\begin{array}{c}\text { Mest. Prof. } \\
19\end{array}$ \\
\hline NORTE - 1 & PA & 1 & UFPA & 1 & & 1 & \\
\hline \multirow{5}{*}{$\begin{array}{c}\text { CENTRO- } \\
\text { OESTE } \\
13\end{array}$} & \multirow[t]{2}{*}{$\mathrm{GO}$} & \multirow[t]{2}{*}{3} & PUC-GOIÁS & 1 & & 1 & \\
\hline & & & UFG & 2 & & 2 & \\
\hline & MS & 1 & UFGD & 1 & & & 1 \\
\hline & DF & 9 & UNB & 9 & 3 & 6 & \\
\hline & & & & Total/Cat. PG & 3 & 9 & 1 \\
\hline \multirow{7}{*}{$\begin{array}{c}\text { NORDESTE } \\
17\end{array}$} & CE & 5 & UFC & 5 & & 5 & \\
\hline & BA & 5 & UFBA & 5 & 3 & 2 & \\
\hline & PB & 1 & UFPB & 1 & & 1 & \\
\hline & $\mathrm{PE}$ & 3 & UFPE & 2 & & 1 & 1 \\
\hline & RN & 2 & UFRN & 2 & 1 & 1 & \\
\hline & SE & 1 & UFS & 1 & & 1 & \\
\hline & & & & Total/Cat. PG & 4 & 12 & 1 \\
\hline \multirow{12}{*}{$\begin{array}{l}\text { SUL } \\
39\end{array}$} & \multirow{3}{*}{ PR } & \multirow{3}{*}{14} & UEL & 2 & & 2 & \\
\hline & & & UEM & 2 & & 2 & \\
\hline & & & UFPR & 10 & & 10 & \\
\hline & \multirow{2}{*}{ SC } & \multirow{2}{*}{8} & UNOCHAPECÓ & 1 & & & 1 \\
\hline & & & UFSC & 7 & & 7 & \\
\hline & \multirow{6}{*}{ RS } & \multirow{6}{*}{17} & UFRGS & 10 & 1 & 9 & \\
\hline & & & FUPF & 2 & & 2 & \\
\hline & & & UFPEL & 1 & & 1 & \\
\hline & & & UFSM & 1 & & 1 & \\
\hline & & & UniRitter & 1 & & 1 & \\
\hline & & & UNISINOS & 2 & & 2 & \\
\hline & & & & Total/Cat. PG & 1 & 37 & 1 \\
\hline \multirow{18}{*}{$\begin{array}{c}\text { SUDESTE } \\
73\end{array}$} & ES & 3 & UFES & 3 & & 3 & \\
\hline & \multirow{5}{*}{ MG } & \multirow{5}{*}{12} & CEFET/MG & 2 & & 2 & \\
\hline & & & UFJF & 2 & & 2 & \\
\hline & & & UFMG & 3 & & 3 & \\
\hline & & & UFOP & 3 & & 2 & 1 \\
\hline & & & UFU & 2 & & 2 & \\
\hline & \multirow{4}{*}{ RJ } & \multirow{4}{*}{19} & UFRJ & 8 & 3 & 3 & 2 \\
\hline & & & UFF & 6 & 1 & 5 & \\
\hline & & & IME & 1 & & 1 & \\
\hline & & & PUC-RIO & 4 & & 4 & \\
\hline & \multirow{7}{*}{ SP } & \multirow{7}{*}{39} & USP & 17 & 4 & 7 & 6 \\
\hline & & & USJT & 2 & & 2 & \\
\hline & & & USP/SC & 1 & 1 & & \\
\hline & & & UNICAMP & 9 & 1 & 8 & \\
\hline & & & UNIP & 1 & 1 & & \\
\hline & & & IPT & 7 & & & 7 \\
\hline & & & UFSCAR & 2 & & 2 & \\
\hline & & & & Total/Cat. PG & 11 & 46 & 16 \\
\hline
\end{tabular}

Fonte: A autora. 


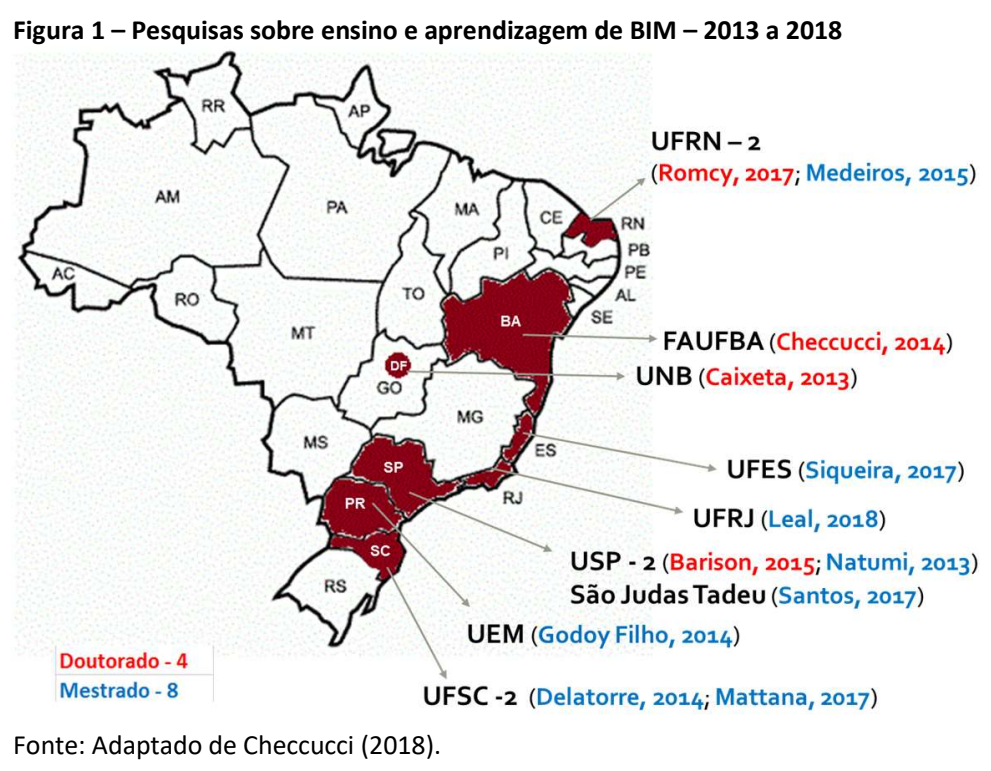

Barison (2015) trata de diversas questões relacionadas com a formação de projetistas, dentre elas: (a) estabelece alguns perfis de formação para especialistas BIM, indicando competências para engenheiros e arquitetos; (b) discute diferentes formas de colaboração para trabalhar BIM no currículo (intracurso, interdisciplinar, à distância e multinacional), além de estratégias para introdução da modelagem nos cursos; e, (c) especifica 3 níveis de proficiência em BIM (introdutório, intermediário e avançado).

Romcy (2017) discute sobre modelagem paramétrica, fabricação digital, projeto e BIM. Apresenta algumas diretrizes - como áreas de conhecimento, conteúdos curriculares e habilidades a serem desenvolvidas - para a adoção de ferramentas e processos paramétricos no ensino de projeto. Dentre as conclusões do trabalho, vale a pena destacar que a autora defende a inserção do projeto paramétrico desde o início do curso e ao longo da formação dos estudantes, de maneira gradual, diluída, e com um aumento crescente de complexidade.

Delatorre (2014), Godoy Filho (2014), Siqueira (2017) e Leal (2018) discutem métodos e diretrizes para inserção de BIM ou de outras Tecnologias de Informação e Comunicação (TICS) no currículo de cursos de graduação.

Delatorre (2014) ressalta que BIM pode potencializar a aprendizagem da arquitetura, mas demanda o desenvolvimento de práticas colaborativas, interdisciplinares e integradas, o que requer mudanças em pessoas e processos. A autora faz uma proposta para adoção do BIM na UNOCHAPECÓ.

Godoy Filho (2014) também identifica no BIM um instrumento importante para o ensino de projeto de arquitetura e defende a adoção de métodos de ensino-aprendizagem ativos, como PBL e PjBL (Problem Based Learning e Project Based Learning).

Siqueira (2017) faz uma proposta para adoção de BIM e Problem Based Learning (PBL) na UFES a curto, médio e longo prazo. Identifica problemas e dificuldades na sua implantação no ensino e propõe procedimentos para superá-los.

Leal (2018) constata que os cursos e professores demandam atualização e apresenta diferentes propostas para inserção nos currículos de TICs como BIM, realidade aumentada, realidade virtual, prototipagem rápida e fabricação digital a partir de experiências já realizadas em práticas pedagógicas divulgadas e outras possibilidades ainda pouco exploradas na academia. 
Medeiros (2015) discute a adoção do BIM para integrar os projetos de arquitetura e estrutura no ensino. Ressalta que o fato de BIM estar sendo trabalhado em uma disciplina obrigatória na Universidade Federal do Rio Grande do Norte deixa os alunos mais confiantes e seguros para utilizar esta modelagem, favorecendo a formação de modeladores BIM nesta universidade.

Já Mattana (2017) discute a adoção de BIM no ensino para trabalhar extração de quantitativos e realizar orçamentação da obra.

Natumi (2013) e Santos (2017) realizam avaliações de experiências de ensinoaprendizagem no Brasil. Natumi (2013) tem uma pesquisa mais ampla sobre o ensino de informática aplicada em cursos de graduação em arquitetura e urbanismo no Brasil e verificou a necessidade de atualização docente. Discute a relevância da adoção de metodologias de ensino-aprendizagem ativas nos cursos de graduação, assim como Checcucci (2014), Godoy Filho (2014) e Siqueira (2017).

Já Santos (2017) tem um trabalho com foco no BIM e analisou matrizes curriculares de cursos de graduação em arquitetura e urbanismo de São Paulo para identificar como esta modelagem estava sendo implantada no estado. Identificou a UNICAMP como a instituição mais avançada naquela época, trabalhando com BIM em pelo menos 5 disciplinas. Verificou também que em São Paulo algumas instituições trabalham BIM, mas ainda sem o desenvolvimento do seu conceito mais amplo.

\section{Discussões}

BIM possui um conceito amplo e complexo que envolve todo o ciclo de vida da edificação e abarca pelo menos 3 diferentes dimensões: tecnológica, processual e humana. Diante disto, são diversas as questões e problemas a serem resolvidos sobre esta modelagem dentre as quais, a título de exemplo, podem ser citadas: as que tratam da sua adoção em empresas e instituições de ensino; do desenvolvimento de padrões e processos de trabalho; da avaliação e aquisição de hardware e software; da capacitação, dimensionamento e contratação de profissionais; do desenvolvimento de métodos e técnicas de trabalho colaborativo e multidisciplinar; da criação de padrões para troca de dados entre diferentes ferramentas; da utilização dos modelos produzidos nas diferentes fases do ciclo de vida do edifício; da integração de BIM com outras TICs, etc.

Considerando as pesquisas agrupadas nas diferentes categorias de análise (Gráfico 2), identificou-se que 57 , ou 39,8\% delas, tratam da etapa de projetação enquanto nas outras categorias (obra; avaliação do BIM; ensino; implantação; FM; BIM+; uso por terceiros e orçamentação) foram encontrados poucos exemplares, entre 6 a 15 trabalhos (que representam de 4,2\% a 10,4\% dos 143 que fazem parte da amostra estudada). Ainda na etapa de projetação, foram encontrados 15 trabalhos que se debruçam sobre a dimensão processual do projeto utilizando BIM, enquanto 14 tratam de questões relacionadas ao conforto e sustentabilidade, como a melhoria do ambiente construído, redução de resíduos, de gasto energético, etc., e 28 trabalhos discutem a aplicação de BIM no Brasil em projetos de alguma disciplina específica, como arquitetura, infraestrutura, estrutura, saneamento, dentre outros (Gráfico 3).

Desta forma observa-se que foram encontrados trabalhos relacionados a todas as fases do ciclo de vida da edificação, ainda que, em alguns casos, em pouca quantidade, como aqueles que discutem FM relacionado com BIM. Das 9 categorias utilizadas para classificação dos trabalhos (projetação; obra; avaliação do BIM; ensino; implantação de BIM; FM; BIM+; uso de BIM por terceiros e orçamentação) foram destacadas aquelas que tratam do ciclo de vida da edificação: projetação (na fase de projeto); orçamentação 
(que pode estar na fase de projeto ou obra e por isto está avaliada independentemente); obra (fase de construção) e FM (fase de uso da edificação). Isto permitiu verificar que 88 trabalhos (ou 62\% dos 143 avaliados) discutem BIM relacionado com alguma fase do ciclo de vida da edificação. 0 Gráfico 4 ilustra esta análise.

Gráfico 4 - avaliação da distribuição dos trabalhos relacionados com o ciclo de vida da edificação

Total de trabalhos avaliados -143

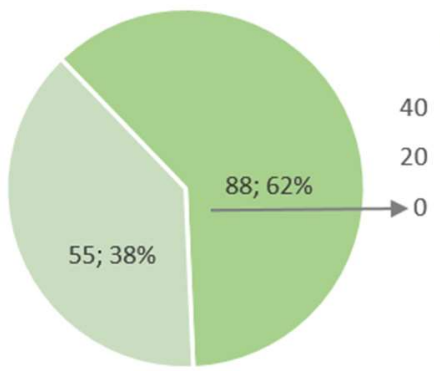

- Trabalhos que tratam do ciclo de vida da edificação - 88 36

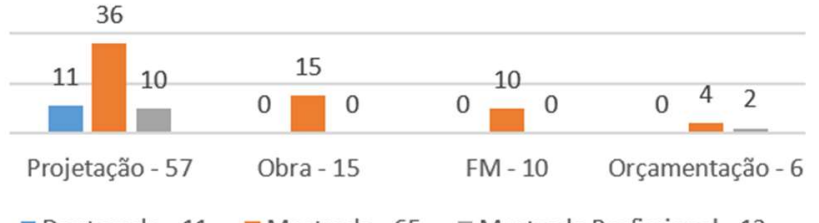

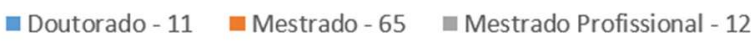

\section{Outras categorias}

(ensino, avaliação, Implantação, uso por terceiros, Bim +) - 55

Fonte: A autora.

Nas universidades, ainda que já existam experiências relevantes de adoção de BIM (CBIC, 2018) percebe-se que são poucas as teses e dissertações que tratam da sua adoção na academia, se considerado todo o país (12 trabalhos ou 8,4\% do total das 143 pesquisas). No entanto, apesar da pequena quantidade de trabalhos encontrados sobre o ensino de BIM, percebeu-se que eles formam um arcabouço com contribuições relevantes que podem, de forma prática, auxiliar na sua implantação nos cursos de graduação do país. Foram encontradas sugestões de diretrizes curriculares, de indicadores para avaliar implantação, conteúdos para disciplinas, propostas de avaliações de estudantes, sugestões de exercícios, de organização curricular, de métodos de ensino-aprendizagem, enfim, um conjunto vasto e diverso de instrumentos que podem ser aplicados para adoção de BIM na formação. Verificou-se a ausência de pesquisas com foco em aplicações ou adoções em cursos de pós-graduação ou tecnólogos, ainda que existam artigos que tratem do tema, como em Cruz, Cuperschmid e Ruschel (2017) e em Rodrigues e Lima (2017).

Chama a atenção que o eixo sul-sudeste do país é responsável por 78,3\% dos trabalhos encontrados (112), enquanto as regiões centro-oeste, nordeste e norte produziram apenas $21,7 \%$ dos trabalhos (31), sendo que apenas 1 deles foi encontrado na região norte do Brasil (na UFPA). A USP foi a instituição que produziu mais trabalhos (17), seguida da UFPR e UFRGS (10 cada) e da UNICAMP e UNB ( 9 cada). No nordeste, vale a pena ressaltar que a UFC e a UFBA produziram 5 trabalhos cada, sendo que nesta última foram desenvolvidas 3 teses e 2 dissertações.

Apenas no Rio Grande do Sul, em Minas Gerais e em São Paulo, foram encontrados trabalhos em mais de 5 instituições de ensino diferentes. Na grande maioria dos estados onde foram encontradas pesquisa sobre BIM, elas foram produzidas por apenas 1 ou 2 instituições, normalmente localizadas em capitais. Isto indica necessidade de interiorização do BIM em todo o país.

Esta constatação evidencia, ainda, que o nível de adoção de BIM no Brasil ainda é inicial e incipiente. Por outro lado, considerando a evolução ano a ano ilustrada no Gráfico 1 é 
possível verificar que o número de pesquisas tem aumentado, indicando crescente interesse da comunidade científica sobre o tema.

Ainda, vale a pena ressaltar que foram encontrados muitos temas interessantes nas pesquisas relacionadas com BIM: sobre sua integração com outras tecnologias; variados exemplos de aplicação em projetos, não apenas de arquitetura mas em outras disciplinas; avaliações diferentes sobre as vantagens da modelagem e a evolução da sua implantação em várias partes do país; propostas e discussão de experiências de adoção por órgãos públicos; uso de BIM para melhorar questões de conforto e sustentabilidade do projeto; dentre outras.

Para ilustrar a variedade dos temas encontrados, as palavras-chave das 19 teses e das 19 dissertações de mestrado profissional foram catalogadas e utilizadas para criar duas nuvens de palavras de forma a mostrar a prevalência e diversidade dos termos (Figuras 2 e 3).

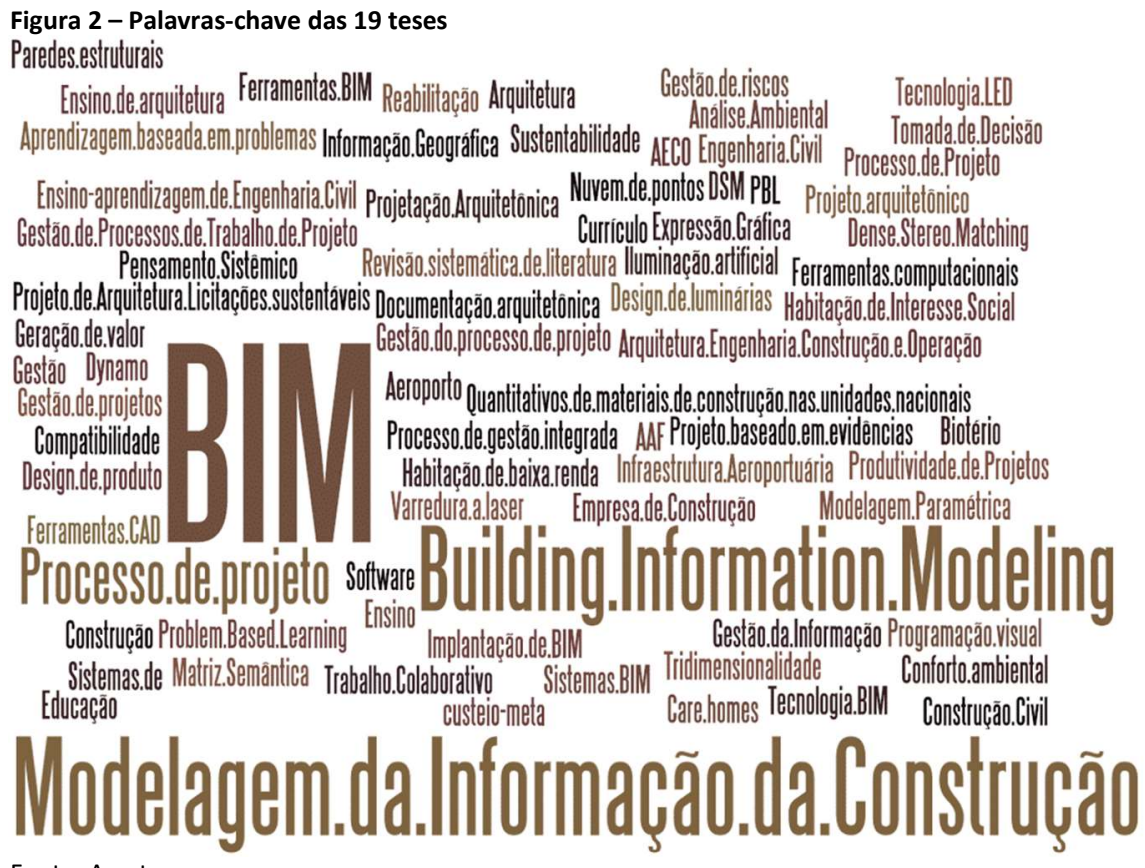

Fonte: A autora.

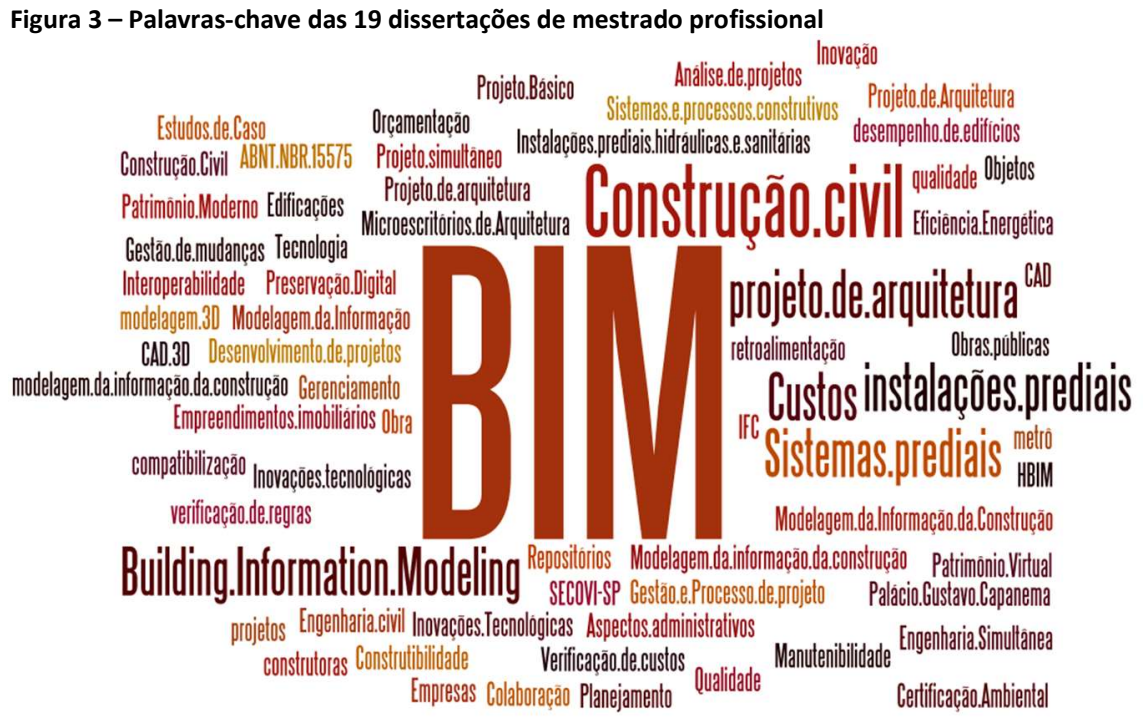

Fonte: A autora. 


\section{Conclusão}

O presente trabalho buscou, selecionou, avaliou, categorizou e analisou teses e dissertações brasileiras publicadas entre 2013 e 2018 no Catálogo de teses e dissertações da CAPES que tratam da Modelagem da Informação da Construção ou Building Information Modeling (BIM).

Foram encontradas 143 pesquisas que discutem o tema, o que representa uma média de mais de 20 trabalhos publicados por ano. Isto indica interesse por parte da comunidade científica para resolver problemas e encaminhar questões relevantes sobre esta modelagem. Ainda, distribuídos os trabalhos nos diferentes anos analisados, percebe-se que o interesse pelo tema vem crescendo.

Dentre os principais resultados encontrados nesta pesquisa pode ser ressaltado que: (a) já existem trabalhos sobre BIM nas diversas fases do ciclo de vida da edificação, mas a maioria deles trata da etapa de projetação; (b) $78,3 \%$ das pesquisas encontradas foram produzidas no eixo sul-sudeste do país e em instituições de ensino sediadas nas capitais e grandes cidades, indicando a necessidade de interiorização e difusão do BIM em outras regiões; (c) existe uma diversidade de temas já pesquisados relacionados com o BIM que encaminham questões sobre a modelagem e podem auxiliar na sua adoção tanto em instituições de ensino quanto no mercado de trabalho.

É importante chamar atenção que este trabalho faz um recorte específico em teses e dissertações já concluídas e entre 2013 e 2018. Uma busca em anais de eventos científicos, que apresenta pesquisas ainda em andamento e uma prospecção nas universidades ampliará bastante o quadro da produção intelectual e das práticas nacionais sobre o tema, haja visto o estudo realizado por Machado, Ruschel e Scheer (2017) e as apresentações que ocorreram no ENEBIM (2018).

Por fim, é importante enfatizar que a maioria das pesquisas identificadas estão gratuitamente disponíveis para acesso no banco de dados da CAPES e formam um conjunto relevante de contribuições que podem alavancar a adoção do BIM no Brasil

\section{Agradecimentos}

A Pró-Reitoria de Pesquisa, Criação e Inovação e Pró-Reitoria de Ensino de Pós-Graduação da Universidade Federal da Bahia pelo apoio através do projeto Jovens Doutores.

\section{Notas}

(1) CAPES. Catálogo de Teses e Dissertações. Disponível em: http://catalogodeteses.capes.gov.br/catalogoteses/\#!/. Acesso em: 10 set. 2018.

(2) A proposta foi realizar uma pesquisa com um recorte temporal dos últimos 5 anos, porém como ela foi realizada entre agosto e setembro de 2018 a autora achou por bem incluir os dados de todos os trabalhos de 2013 o que incorporou na amostra trabalhos publicados entre janeiro de 2013 a setembro de 2018.

(3) Esta seção do trabalho foi apresentada de forma resumida no ENEBIM (2018) e ampliada para ser publicada na revista.

\section{Referências}

ANDRADE, M. L. V. X; RUSCHEL, R. C. BIM: conceitos, cenários das pesquisas publicadas no Brasil e tendências. In: SIMPÓSIO BRASILEIRO DE QUALIDADE DE PROJETOS, 1., 2009, São Carlos. Anais [...]. São Carlos: RiMa, 2009.

BARDIN, L. Análise de conteúdo. São Paulo: Edições 70; Persona, 1979. 229 p. 
BARISON, M. B. Introdução de modelagem da informação da construção (BIM) no currículo: uma contribuição para a formação do projetista. 2015. 390 f. Tese (Doutorado em engenharia civil) - Universidade de São Paulo, São Paulo, 2015.

BERNSTEIN, H. M. et al. The Business Value of BIM for Construction in Major Global Markets. Bedford: McGraw Hill Construction, 2014.

BRASIL. Decreto n. 9.377, de 17 de maio de 2018. Institui a Estratégia de Disseminação do Building Information Modelling. Diário Oficial da União, Brasília, Edição 95, Seção 1, p. 3, mai. 2018. Atos do Poder Executivo. Disponível em: http://www.planalto.gov.br/ccivil_03/_at02015-2018/2018/Decreto/D9377.htm. Acesso em: 5 out. 2018.

CAIXETA, Luciano Mendes. Estudo crítico sobre o uso de ferramentas de modelagens tridimensionais de informações digitais BIM no ensino contemporâneo da arquitetura. 2013. 175 f. Tese (Doutorado em arquitetura e urbanismo) - Universidade de Brasília, Brasília, 2013.

CBIC. Câmara Brasileira da Indústria da Construção. Encontro Nacional sobre o Ensino de BIM impacta participantes com discussões e experiências compartilhadas. Disponível em: https://cbic.org.br/encontro-nacionalsobre-o-ensino-de-bim-impacta-participantes-com-discussoes-e-experiencias-compartilhadas/. Acesso em: 15 out. 2018.

CHECCUCCI, E. S. Ensino-aprendizagem de BIM nos cursos de graduação em Engenharia Civil e o papel da Expressão Gráfica neste contexto. 2014. 17 f. il. Tese (Doutorado em Difusão do Conhecimento) - Faculdade de Educação, Universidade Federal da Bahia, Salvador, 2014.

CHECCUCCI, E. S. Pesquisas de pós-graduação brasileiras sobre ensino e aprendizagem de BIM. In: ENCONTRO NACIONAL SOBRE O ENSINO DE BIM, 1., Campinas, 2018. Anais [...] Campinas: ANTAC, 2018.Disponível em: https://www.antaceventos.net.br/index.php/enebim/2018/paper/view/68. Acesso em: 5 out. 2018.

CHECCUCCI, E. de S.; AMORIM, A. L. Método para análise de componentes curriculares: identificando interfaces entre um curso de graduação e BIM. PARC Pesquisa em Arquitetura e Construção, Campinas, v. 5, n. 1, p. 6-17, jan./jun. 2014. Disponível em: https://periodicos.sbu.unicamp.br/ojs/index.php/parc/article/view/8634540/2461. Acesso em: 15 ago. 2016.

CHECCUCCI, E. S.; PEREIRA, A. P. C.; AMORIM, A. L. A Difusão das Tecnologias BIM por Pesquisadores do Brasil. In: ENCONTRO NACIONAL DE TECNOLOGIA DE INFORMAÇÃO E COMUNICAÇÃO NA CONSTRUÇÃO CIVIL, 5., Salvador, 2011. Anais [...]. Salvador: LCAD/PPGAU - UFBA, 2011.

CHECCUCCI, E. S.; PEREIRA, A. P. C.; AMORIM, A. L. Uma visão da difusão e apropriação do paradigma BIM no Brasil - TIC 2011. Gestão e Tecnologia de Projetos, São Paulo, v. 8, n. 1, p. 19-39, jan.-jun. 2013. DOI:

http://dx.doi.org/10.4237/gtp.v8i1.232. Acesso em: 15 ago. 2016.

CRUZ, M. O.; CUPERSCHMID, A.; RUSCHEL, R. C. A incorporação de BIM no ensino do curso Técnico em Edificações. Gestão \& Tecnologia de Projetos, São Carlos, v. 12, n. 2, p. 117-134. 2017. DOI:

http://dx.doi.org/10.11606/gtp.v12i2.131498. Acesso em: 15 out. 2018.

DELATORRE, V. Potencialidades e limites do BIM no ensino de arquitetura: uma proposta de implementação. 2014. 293 f. Dissertação (Mestrado em arquitetura e urbanismo) - Centro Tecnológico, Universidade Federal de Santa Catarina, Florianópolis, 2014.

ENEBIM. ENCONTRO NACIONAL SOBRE O ENSINO DE BIM, 1., Campinas. Anais [...]. Campinas: ANTAC, 2018. Disponível em: http://www.antaceventos.net.br/index.php/enebim/2018/schedConf/presentations. Acesso em: 5 out. 2018.

ITO, A. L. Y.; SCHEER, S. Um levantamento em Curitiba das percepções do potencial do BIM no ensino em cursos de arquitetura. SIMPÓSIO BRASILEIRO DE TECNOLOGIA DA INFORMAÇÃO E COMUNICAÇÃO NA CONSTRUÇÃO, 1., SIMPÓSIO BRASILEIRO DE GESTÃO E ECONOMIA DA CONSTRUÇÃO, 10., 2017, Fortaleza, Brasil. Anais [...]. Fortaleza, 
CE: Marketing Aumentado, 2017. Disponível em: https://marketingaumentado.com.br/sbtic/files/2017/paper_135.pdf. Acesso em: 27 set. 2018.

$\mathrm{KIM}$, J. Use of BIM for effective visualization teaching approach in construction education. Journal of professional issues in engineering education and practice. ASCE, v. 138, n. 3, p. 214-223, 2012. Disponível em: http://www.ascelibrary.org. Acesso em: 20 set. 2018.

KYMMELL, W. Building information modeling: Planning and managing construction projects with 4D CAD and simulations. Nova York: McGraw Hill, 2008. 270 p.

LEAL, B. M. F. Propostas para o ensino dos conteúdos de arquitetura e urbanismo através de ferramentas digitais. 2018. 174 f. Dissertação (Mestrado em arquitetura) - Universidade Federal do Rio de Janeiro, Rio de Janeiro, 2018.

MACEDO, R. S. Outras Luzes: um rigor intercrítico para uma etnopesquisa política. In: MACEDO, R. S.; GALEFFI, D. A; PIMENTEL, A. Um rigor outro. Sobre a questão da qualidade na pesquisa qualitativa. Salvador: EDUFBA, 2009. p. 75126.

MACHADO, F.; RUSCHEL, R. C.; SCHEER, S. Análise da produção científica brasileira sobre a Modelagem da Informação da Construção. Ambient. constr. [online]. Porto Alegre, v. 17, n. 4, p. 359-384, out. /dez. 2017. DOI: http://dx.doi.org/10.1590/s1678-86212. Acesso em: 5 out. 2018.

KASSEM, M. Assessing and improving Market BIM Maturity: Conceptual Constructs and Practical Tools. In: ENCONTRO NACIONAL SOBRE O ENSINO DE BIM, 1., 2018, Campinas. Anais [...]. Campinas: ANTAC, 2018. Disponível em: http://www.antaceventos.net.br/index.php/ enebim/2018/paper/view 017000400202. Acesso em: 2 fev. 2019.

MARCONI, M. de A.; LAKATO, E. M. Fundamento de metodologia científica. 5. ed. São Paulo: Editora Atlas S. A., 2003.

MEDEIROS, S. C. S. Integração de Projeto de Arquitetura e Estruturas no ensino através de BIM: uma abordagem dos cursos de arquitetura e urbanismo da UFRN e da UFPB. 2015. 95 f. Dissertação (Mestrado em arquitetura e urbanismo) - Universidade Federal do Rio Grande do Norte, Natal, 2015.

MATTANA, L. Contribuição para o ensino de orçamentação com uso de BIM no levantamento de quantitativos. 2017. 279 f. Dissertação (Mestrado em arquitetura e urbanismo) - Universidade Federal de Santa Catarina, Florianópolis, 2017.

MOTA, P. P.; RUSCHEL, R. C. Identificação do conhecimento acadêmico de Bim integrado ao gerenciamento de facilities. In: ENCONTRO NACIONAL DE TECNOLOGIA DO AMBIENTE CONSTRUÍDO, 16., 2016. São Paulo, Brasil. Anais [...]. Disponível em: http://www.infohab.org.br/entac/2016/ENTAC2016_paper_272.pdf. Acesso em: 28 set. 2018 p.4951-4962.

MOTA, P. P.; RUSCHEL, R. C. Caracterização de modelos BIM com foco em gestão de ativos. In: SIMPÓSIO BRASILEIRO DE TECNOLOGIA DA INFORMAÇÃO E COMUNICAÇÃO NA CONSTRUÇÃO, 1., SIMPÓSIO BRASILEIRO DE GESTÃO E ECONOMIA DA CONSTRUÇÃO, 10., 2017, Fortaleza, Brasil. Anais [...]. Fortaleza, CE: Marketing Aumentado, 2017.

NATUMI, Y. O ensino de informática aplicada nos cursos de graduação em arquitetura e urbanismo no Brasil. 2013. 282 f. Dissertação (Mestrado em arquitetura e urbanismo) - Universidade de São Paulo, São Paulo, 2013.

RODRIGUES; LIMA, 2017. Mapeamento de interfaces entre disciplinas de Engenharia Civil e o paradigma BIM. In: SIMPÓSIO BRASILEIRO DE TECNOLOGIA DA INFORMAÇÃO E COMUNICAÇÃO, 1.; SIMPÓSIO BRASILEIRO DE GESTÃO E ECONOMIA DA CONSTRUÇÃO, 10., 2017, Ceará, Brasil. Anais [...]. Fortaleza: UFC, 2017

ROMCY, N. M. S. Abordagem paramétrica e ensino de projeto: Proposição de diretrizes metodológicas, considerando estratégias curriculares e o atelier de projeto. 2017. 317 f. Tese (Doutorados em arquitetura e urbanismo) - Universidade Federal do Rio Grande do Norte, Natal, 2017. 
RUSCHEL, R. C.; ANDRADE, M. L. V. X.; SALES, A. A.; MORAIS, M. O ensino de BIM: exemplos de implantação em cursos de engenharia e arquitetura. In: SIMPÓSIO BRASILEIRO DE TECNOLOGIA DA INFORMAÇÃO E COMUNICAÇÃO NA CONSTRUÇÃO, 1., SIMPÓSIO BRASILEIRO DE GESTÃO E ECONOMIA DA CONSTRUÇÃO, 10., 2017, Fortaleza, Brasil. Anais [...]. Fortaleza, CE: Marketing Aumentado, 2017.

RUSCHEL, R. C.; ANDRADE, M. L. V. X.; MORAIS, M. O ensino de BIM no Brasil: onde estamos? Ambient. constr. [online]. Porto Alegre, 2013, vol. 13, n. 2, pp. 151-165. ISSN 1678-8621. DOI: http://dx.doi.org/10.1590/S167886212013000200012. Acesso em: 20 mar. 2014.

SANTOS, D. V.; LIMA, M. M. X.; CAMPOS, V. R. Análise da percepção do uso do BIM em diferentes implantações: estudo de caso. In: ENCONTRO DE TECNOLOGIA DE INFORMAÇÃO E COMUNICAÇÃO NA CONSTRUÇÃO, 5., 2011, Salvador, Brasil. Anais [...]. Salvador: UFBA, 2011. 1 CD-ROM.

SANTOS, L. A. Building information modeling no ensino de arquitetura e urbanismo: Percepção e disseminação do BIM nas Instituições de Ensino Superior do Estado de São Paulo. 2017. 138 f. Dissertação (Mestrado em arquitetura e urbanismo) - Universidade São Judas Tadeu, São Paulo, 2017.

SCHEER, S.; AYRES FILHO, C. Abordando a BIM em níveis de modelagem. In: WORKSHOP BRASILEIRO DE GESTÃO DO PROCESSO DE PROJETO NA CONSTRUÇÃO DE EDIFÍCIOS, 9., São Carlos, Brasil. Anais [...]. São Paulo: USP, 2009. 1 CD-ROM.

SIQUEIRA, L. S. R. Aplicação das metodologias building information modeling (BIM) e aprendizagem baseada em problemas (ABP) no curso de graduação em engenharia civil / UFES: diagnóstico e recomendações. 2017.138 f. Dissertação (Mestrado em engenharia civil) - Universidade Federal do Espírito Santo, Vitória, 2017.

SUCCAR, B. Building information modeling framework: A research and delivery foundation for industry stakeholders. Automation in Construction. [S.I.], v. 18, p. 357-375, 2009. DOI:

https://doi.org/10.1016/j.autcon.2008.10.003. Acesso em: 6 fev. 2010.

SUCCAR, B.; KASSEM, M. Macro-BIM adoption: Conceptual structures. Automation in Construction, [S.I.], v. 57, p. 64-79, 2015. DOI: https://doi.org/10.1016/j.autcon.2015.04.018. Acesso em: 5 out. 2018.

\section{1 Érica de Sousa Checcucci}

Arquiteta. Doutora. Professora na Universidade Federal da Bahia. Endereço postal: Rua Caetano Moura, Número 121, Federação, Salvador, Ba, Brasil, CEP. 40210-905. 